\title{
Immunogenicity and Safety of a Fully Liquid DTaP-IPV-Hep B-PRP-T Vaccine at 2-4-6 Months of Age in Peru
}

\section{Claudio Lanata1 ${ }^{1}$, Betzana Zambrano ${ }^{2}$, Lucie Ecker ${ }^{1}$, Isabel Amemiya ${ }^{1}$, Ana Gil ${ }^{1}$ and Eduardo Santos Lima ${ }^{3 *}$}

1 Instituto de Investigación Nutricional, Lima, Peru

${ }^{2}$ Sanofi Pasteur, Montevideo, Uruguay

${ }^{3}$ Clinical Development, Sanofi Pasteur, Lyon, France

\begin{abstract}
Objectives: To assess the immunogenicity and safety of a new candidate, fully liquid, hexavalent DTaP-IPVHep B-PRP-T vaccine (Hexaxim ${ }^{\mathrm{TM}}$, an AcXim family vaccine) compared to a licensed hexavalent DTaP-IPV-Hep B// PRP-T vaccine (Infanrix hexa ${ }^{\circledR}$ ) in Peru.
\end{abstract}

Methods: Infants born to HBsAg seronegative mothers and who had not received a hepatitis B vaccine prior to entry into the study were randomized to receive either Hexaxim ${ }^{\text {TM }}$ (Group 1) or Infanrix hexa ${ }^{\circledR}$ (Group 2) at 2, 4, 6 months of age. Seroprotection (SP) rate for hepatitis B (anti-HBs antibody concentration $\geq 10 \mathrm{mlU} / \mathrm{mL}$ ) was analysed for non-inferiority (Group 1 minus Group 2) 1 month post-primary series. Anti-diphtheria and anti-polyrosil ribitol phosphate (PRP) antibody responses were analysed descriptively. Safety was analysed from parental reports.

Results: Seroprotection rate for anti-HBs antibody titers $\geq 10 \mathrm{mlU} / \mathrm{mL}$ was high in both groups $(\geq 99.2 \%)$ and non-inferiority was demonstrated (lower bound of the $95 \% \mathrm{Cl}$ for the difference was -4.17 , above the pre-defined delta [-10\%]). Post-primary SP rates for anti-diphtheria ( $\geq 95.5 \% \geq 0.01 \mathrm{IU} / \mathrm{mL})$, anti-PRP $(\geq 99.2 \% \geq 0.15 \mu \mathrm{g} / \mathrm{mL})$, and anti-HBs $\geq 100 \mathrm{mlU} / \mathrm{mL}(\geq 93.9 \%)$, were similar in each group. Both vaccines were well tolerated. The incidence of serious adverse avents was low and similar in each group, and none was considered to be vaccine related.

Conclusions: In a 2, 4, 6 month schedule in Peruvian infants, the investigational DTaP-IPV-Hep B-PRP-T fully liquid vaccine provided high immunogenicity for $\mathrm{HBs}$, diphtheria and PRP vaccine antigens that was comparable to the licensed hexavalent vaccine. Both vaccines had a similar safety profile.

Keywords: Vaccine; Hexavalent; Pediatric; Combination; Immunization

\section{Introduction}

The investigational hexavalent vaccine $\left(\right.$ Hexaxim $\left.^{\mathrm{TM}}\right)$ is fully liquid and part of Sanofi Pasteur's AcXim family, which includes the established tetra- and pentavalent vaccines Tetraxim $^{\circledR} /$ Tetravac $^{\circledR}$ and Pentaxim $^{\circledR} /$ Pentavac $^{\circledR}$. It combines a new Hansenula polymorphaderived and thimerosal-free hepatitis B (Hep B) antigen [1-3] with well-established diphtheria toxoid (D), tetanus toxoid (T), acellular (2-component [pertussis toxoid (PT) and filamentous hemagglutinin (FHA)]) pertussis (aP), inactivated poliovirus (IPV), and Haemophilus influenzae type b polysaccharide conjugated to tetanus protein (PRP-T) antigens to produce a new hexavalent vaccine. The IPV and PRP-T antigens are WHO pre-qualified [4] as standalone vaccines (Imovax ${ }^{\mathbb{R}}$ Polio and ActHib ${ }^{\circledR}$, respectively). The immunogenicity and safety of the new Hep B antigen have been described following both monovalent administration to adults and adolescents [5] as well as following administration of the new hexavalent vaccine in several pediatric clinical studies in a range of ethnic populations and administration schedules [6-8].

Accepted advantages of combination vaccines include a reduced number of injections coupled with increased compliance to increasingly challenging pediatric vaccination schedules, leading to improved disease control with associated reduced direct and indirect costs [9]. The routine use of combination vaccines has been crucial in reducing the incidence of childhood diseases [9]. However, regional disparities remain, such as the use of combination vaccines based on aP versus whole-cell pertussis $(\mathrm{wP})$ vaccines or the inclusion or not of hepatitis B and/or IPV versus the use of a standalone hepatitis B vaccine and/or the oral poliovirus vaccine (OPV). The investigational vaccine, including acP and IPV valences, aims to provide protection against six diseases that are considered by WHO to be priorities and is presented in a ready-to-use, fully liquid formulation.

Being a fully liquid vaccine, Hexaxim minimizes human error associated with vaccine re-constitution, and helps to improve vaccination compliance. But more importantly, the introduction of a second hexavalent vaccine against D, T, P, IPV, hepatitis B, and Hib, will be vital in the event of global production and supply ruptures, which can arise intermittently even when established, well-monitored and controlled processes are in place. In such instances, the availability of a second hexavalent vaccine will mean that vaccination coverage rates could be more easily maintained globally, minimising potential outbreaks of these six pediatric infectious diseases.

This clinical study was performed to document immunogenicity and safety data in a Peruvian pediatric population following administration of the investigational hexavalent vaccine at 2, 4 and 6

*Corresponding author: Eduardo Santos-Lima, Director, Clinical Development, Sanofi Pasteur, 1541 avenue, Marcel Mérieux, 69280 Marcy l'Etoile, France, Tel: +33 (0) 4373758 53; Fax: + 33 (0) 437377888 E-mail: eduardo.santos-lima@sanofipasteur.com

Received January 10, 2012; Accepted March 12, 2012; Published March 18, 2012

Citation: Lanata C, Zambrano B, Ecker L, Amemiya I, Gil A, et al. (2012) Immunogenicity and Safety of a Fully Liquid DTaP-IPV-Hep B-PRP-T Vaccine at 2-4-6 Months of Age in Peru. J Vaccines Vaccin 3:128. doi:10.4172/21577560.1000128

Copyright: ( 2012 Lanata C, et al. This is an open-access article distributed unde the terms of the Creative Commons Attribution License, which permits unrestricted use, distribution, and reproduction in any medium, provided the original author and source are credited. 
months of age. As the Hep B antigen is the novel component of the investigational vaccine, the non-inferiority of Hep B seroprotection (SP) compared to a licensed vaccine comparator was the primary objective of this study, the immunogenicity of the other vaccine components having been demonstrated in previous studies with this [6-8] and other AcXim family vaccines that include some of the same antigens [10] - for the two-component aP antigen, a recently published review concluded high and similar immunogenicity irrespective of the study population and immunization schedule [11]. In our study, we also included the immunogenicity of the PRP antigen to compare the response following its administration as a valence of the fully liquid, investigational vaccine to its administration as a lyophilized constituent of the comparator vaccine. Finally, we describe the immunogenicity of the $\mathrm{D}$ antigen and include anti-D concentration data prior to the first dose (as well as after the final dose) to provide an indication of the level of passively transmitted maternal anti-D antibodies and their effect on the post-primary series response.

\section{Materials and Methods}

\section{Study design and participants}

This was a Phase III, observer-blind, randomized, controlled study performed in a single centre in Peru (ClinicalTrials.gov identifier: NCT00831753). Healthy 2-month old infants who were born at fullterm ( $\geq 37$ weeks) and with birth weight $\geq 2.5 \mathrm{~kg}$ were included in the study, which took place between May 2008 and May 2009. Mothers were screened for hepatitis B surface antigen (HBsAg) in either the last 30 days of pregnancy ( $\geq 36$ weeks of amenorrhea) or in the first 30 days post-partum; only babies born to HBsAg-negative mothers were considered for inclusion in the study. In addition, no hepatitis B vaccine was to have been administered prior to entry into the study at 2 months of age.

Other criteria for exclusion were if the infant was febrile (temperature $>38.0^{\circ} \mathrm{C}$ ), had taken part in or was planning to take part in a clinical study in parallel, was immunodeficient, had received any blood-derived product since birth, or if any illness contraindicated study inclusion. In addition, infants were not included if any vaccination had been administered in the 4 weeks prior to the first study vaccination, if any vaccination was planned during the study (other than the study vaccines, rotavirus and pneumococcal conjugate vaccines), or if maternal HIV or hepatitis $\mathrm{C}$ seropositivity was known.

Following inclusion in the study, a randomization list created under the responsibility of Sanofi Pasteur's statistics department was used to assign infants to receive either the investigational DTaP-IPVHep B-PRP-T vaccine (Group 1) or the licensed DTaP-IPV-Hep B// PRP-T comparator (Group 2).

The protocol and informed consent form and their amendments were approved by the independent ethics committees of the study centre (Instituto de Investigación Nutricional, Lima, Peru) and by the Peruvian Ministry of Health. The study was done in accordance with the recommendations of the Declaration of Helsinki (Edinburgh revision, October 2000) and International Conference on Harmonisation (ICH) Good Clinical Practice (GCP), and with applicable national and local requirements.

\section{Vaccines and vaccine administration}

The investigational DTaP-IPV-Hep B-PRP-T vaccine (batch number S4009F04) (Hexaxim $^{\mathrm{TM}}$ ) was manufactured by Sanofi Pasteur and supplied in pre-filled $0.5 \mathrm{~mL}$ syringes that were shaken gently before injection. The needle size was $25 \mathrm{G} / 16 \mathrm{~mm}$. Each $0.5 \mathrm{~mL}$ dose was preservative-free and contained $\geq 20$ IU diphtheria toxoid, $\geq 40$ IU tetanus toxoid, $25 \mu \mathrm{g}$ pertussis toxoid (PT), $25 \mu \mathrm{g}$ filamentous hemagglutinin (FHA), 40, 8 and 32 D-antigen units of IPV type 1, 2 and 3, respectively, $12 \mu \mathrm{g}$ of Hemophilus influenzae type b polysaccharide conjugated to tetanus toxoid, $10 \mu \mathrm{g} \mathrm{HBsAg}$, and $0.6 \mathrm{mg}$ aluminum hydroxide (total aluminum content of $0.6 \mathrm{mg}$ per dose).

The control DTaP-IPV-Hep B//PRP-T vaccine (batch number A21CA310C) (Infanrix hexa ${ }^{\circledR}$ ) was manufactured by GlaxoSmithKline Biologicals and supplied as two separate components (a DTaP-Hep B-IPV suspension in a pre-filled syringe and lyophilized PRP-T as a white pellet in a glass vial) that were reconstituted as a 0.5 $\mathrm{mL}$ dose immediately prior to injection. The needle size was $25 \mathrm{G} / 25$ $\mathrm{mm}$. Each dose contained 2-phenoxyethanol as preservative, $\geq 30$ IU of diphtheria toxoid, 40 IU tetanus toxoid, $25 \mu \mathrm{g}$ PT, $25 \mu \mathrm{g}$ FHA, $8 \mu \mathrm{g}$ pertactin, 40, 8 and $32 \mathrm{D}$-antigen units of IPV type 1, 2 and 3 , respectively, $10 \mu \mathrm{g}$ of Hemophilus influenzae type $\mathrm{b}$ polysaccharide conjugated to 20-40 $\mu$ g tetanus toxoid, $10 \mu \mathrm{g}$ HBsAg; the PRP-T and HBsAg were adsorbed on $1.45 \mathrm{mg}$ aluminum phosphate and the $\mathrm{D}, \mathrm{T}$, PT, FHA and pertactin were adsorbed on $0.95 \mathrm{mg}$ hydrated aluminum oxide (total aluminum content of $0.8 \mathrm{mg}$ per dose).

The investigational and control vaccines were administered intramuscularly into the anterolateral aspect of the-preferably rightthigh.

\section{Serology}

A $4 \mathrm{~mL}$ blood sample was taken at 2 months of age (i.e. prior to the first primary series vaccination) for determination of anti-D antibody concentration and a $5 \mathrm{~mL}$ sample was taken at 7 months of age (i.e. 1 month after the third vaccination) for assessment of antibodies to anti-D, anti-PRP and anti-Hep B. We focused on these three antigens for the reasons described in the Introduction, and elucidated in the Discussion.

Serological analyses were done at the Sanofi Pasteur Global Clinical Immunology Laboratory in the USA. Anti-Hep B antibody concentrations were measured using the commercially-available VITROS $^{\circledR}$ anti-HBs assay (Ortho-Clinical Diagnostics, Inc.); anti-D antibody concentrations were measured by a toxin neutralization test and anti-PRP antibody concentrations were measured by a Farr-type radioimmunoassay (RIA).

\section{Reactogenicity and safety}

For routine evaluation of reactogenicity (pre-defined [solicited] adverse events) and safety, each participant was observed by the Investigator for 30 minutes after each vaccination to monitor for any immediate adverse events. In addition, for solicited injection site (pain, erythema, and swelling) and systemic (pyrexia, vomiting, crying, somnolence, anorexia, and irritability) reactions ${ }^{1}$, daily intensity/ measurement was recorded by the parent(s)/legally responsible representative using diary cards for 7 days following each vaccination (and pertinent details if ongoing after that time). The parent(s)/legally responsible representative also recorded the start/stop date, intensity/ measurement and other pertinent details of any non-solicited events to the next visit. All non-solicited injection site events were considered to be related to the vaccination and so were recorded as non-solicited injection site reactions; the relationship to the vaccination for non-

${ }^{1}$ All solicited events were considered to be related to the vaccination, and any event related to vaccination was termed a 'reaction'. 
solicited systemic events was assessed by the Investigator. Each adverse event was categorized as Grade 1 (mild), Grade 2 (moderate) or Grade

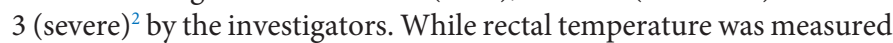
at study visits by the Investigator, axillary rather than rectal temperature was measured by the parent(s)/legally responsible representative between visits for cultural and compliance reasons. No temperature conversion calculation was made for the route of measurement used.

Serious adverse events (SAEs) were collected throughout the study, until 6 months after the final primary series vaccination.

\section{Statistical analyses}

The primary objective of the study was to demonstrate noninferiority for Hep B SP rate, based on a threshold of $10 \mathrm{mIU} / \mathrm{mL}$, at 1 month after the three-dose primary vaccination series, for the investigational DTaP-IPV-Hep B-PRP-T vaccine compared to the licensed DTaP-IPV-Hep B//PRP-T comparator. The non-inferiority comparison was based on the difference (Group 1 minus Group 2) in SP rate for anti-Hep B antibody concentrations $\geq 10 \mathrm{mIU} / \mathrm{mL}$, with non-inferiority being concluded if the lower bound of the two-sided $95 \%$ confidence interval (CI) of the difference was above $-10 \%$. The 95\% CI for the difference was calculated based on the Wilson score method without continuity correction as described by Newcombe [12].

Secondary objectives included further description of the immunogenicity of the Hep B antigen, as well as the D and PRP antigens for both the investigational and control vaccines. Geometric mean antibody concentrations were calculated with their 95\% CIs using the normal approximation method, and the percentages of participants achieving pre-defined thresholds (Table 1) were calculated with their 95\% CIs using the exact binomial distribution for percentages (ClopperPearson method, quoted by Newcombe [13]). Additionally, safety was analysed as a secondary objective in each group (\% of participants with a particular event and associated 95\% CI calculated using the exact binomial distribution for percentages) (Clopper-Pearson method, quoted by Newcombe [13]).

It was planned to include 266 participants (133 in each group); this sample size calculation was done using the Farrington and Manning formula [14] based on type 1 error of $2.5 \%$ (one-sided hypothesis) to provide an

overall power of $90 \%$ for the primary objective, assuming a SP rate of $96 \%$ and an attrition rate of $15 \%$.

The Intent to Treat (ITT) analysis set comprised all participants who received at least one dose of vaccine, analysed by randomisation group. The Per Protocol (PP) analysis set comprised ITT participants who received the three doses of primary series with no protocol deviations. The Safety Analysis Set comprised participants who received at least one dose of study vaccine, analysed by vaccine received. The primary hypothesis of non-inferiority for Group 1 minus Group 2 immunogenicity was tested on the PP analysis set and confirmed using the ITT analysis set. The Safety Analysis Set was used for the safety analysis.

All analyses were done using SAS software, Version 9.1 (SAS Institute, Cary, NC, USA).

\begin{tabular}{|c|c|c|c|c|c|c|c|c|}
\hline \multirow[b]{2}{*}{ Antibody } & \multirow[b]{2}{*}{ Timepoint } & \multirow[b]{2}{*}{ Endpoint } & \multicolumn{2}{|c|}{ Group $1(\mathrm{~N}=132)$} & \multicolumn{2}{|c|}{ Group $2(\mathrm{~N}=130)$} & \multicolumn{2}{|c|}{ Group 1 minus Group 2} \\
\hline & & & $\begin{array}{l}\% \text { or GMC or } \\
\log _{10} \mathrm{GMC}\end{array}$ & $\begin{array}{l}(95 \% \mathrm{Cl}) \text { or } \\
\log _{10}(\mathrm{SD})\end{array}$ & $\begin{array}{l}\% \text { or GMC or } \\
\log _{10} \mathrm{GMC}\end{array}$ & $\begin{array}{l}(95 \% \mathrm{Cl}) \text { or } \\
\log _{10}(\mathrm{SD})\end{array}$ & $\%$ & $(95 \% \mathrm{Cl})$ \\
\hline \multicolumn{9}{|c|}{ Primary objective: } \\
\hline Anti-Hep B & Post-Dose 3 & $\geq 10 \mathrm{mlU} / \mathrm{mL}$ & $99.2 \%$ & $(95.9 ; 100.0)$ & $100.0 \%$ & $(97.2 ; 100.0)$ & -0.76 & $(-4.17 ; 2.18)$ \\
\hline \multicolumn{9}{|c|}{ Secondary objectives: } \\
\hline \multirow[t]{3}{*}{ Anti-Hep B } & Post-Dose 3 & $\geq 100 \mathrm{mIU} / \mathrm{mL}$ & $93.9 \%$ & $(88.4 ; 97.3)$ & $99.2 \%$ & $(95.8 ; 100.0)$ & NC & NC \\
\hline & & GMC (IU/mL) & 986 & $(764 ; 1270)$ & 1139 & $(961 ; 1350)$ & NC & NC \\
\hline & & $\log _{10} \mathrm{GMC}$ & 2.99 & $(0.641)$ & 3.06 & $(0.424)$ & NC & NC \\
\hline \multirow[t]{4}{*}{ Anti-PRP } & Post-Dose 3 & $\geq 0.15 \mu \mathrm{g} / \mathrm{mL}$ & $100.0 \%$ & $(97.2 ; 100.0)$ & $99.2 \%$ & $(95.8 ; 100.0)$ & NC & NC \\
\hline & & $\geq 1 \mu \mathrm{g} / \mathrm{mL}$ & $84.8 \%$ & $(77.6 ; 90.5)$ & $83.8 \%$ & $(76.4 ; 89.7)$ & NC & NC \\
\hline & & $\mathrm{GMC}(\mu \mathrm{g} / \mathrm{mL})$ & 5.22 & $(4.04 ; 6.73)$ & 3.93 & $(3.17 ; 4.89)$ & NC & NC \\
\hline & & $\log _{10} \mathrm{GMC}$ & 0.718 & $(0.642)$ & 0.595 & $(0.543)$ & NC & NC \\
\hline \multirow[t]{8}{*}{ Anti-D } & Pre-Dose 1 & $\geq 0.01 \mathrm{IU} / \mathrm{mL}$ & $82.6 \%$ & $(75.0 ; 88.6)$ & $84.6 \%$ & $(77.2 ; 90.3)$ & NC & $\mathrm{NC}$ \\
\hline & & $\geq 0.1 \mathrm{IU} / \mathrm{mL}$ & $66.7 \%$ & $(57.9 ; 74.6)$ & $70.0 \%$ & $(61.3 ; 77.7)$ & NC & NC \\
\hline & & GMC (IU/mL) & 0.183 & $(0.122 ; 0.277)$ & 0.276 & $(0.178 ; 0.427)$ & NC & $\mathrm{NC}$ \\
\hline & & $\log _{10} \mathrm{GMC}$ & -0.737 & (1.04) & -0.560 & (1.10) & NC & $\mathrm{NC}$ \\
\hline & Post-Dose 3 & $\geq 0.01 \mathrm{IU} / \mathrm{mL}$ & $95.5 \%$ & $(90.4 ; 98.3)$ & $100.0 \%$ & $(97.2 ; 100.0)$ & NC & NC \\
\hline & & $0.1 \mathrm{IU} / \mathrm{mL}$ & $58.3 \%$ & $(49.4 ; 66.8)$ & $65.4 \%$ & $(56.5 ; 73.5)$ & NC & $\mathrm{NC}$ \\
\hline & & GMC (IU/mL) & 0.156 & $(0.119 ; 0.204)$ & 0.192 & $(0.154 ; 0.239)$ & NC & $\mathrm{NC}$ \\
\hline & & $\log _{10} \mathrm{GMC}$ & -0.807 & $(0.680)$ & -0.717 & $(0.554)$ & NC & $\mathrm{NC}$ \\
\hline
\end{tabular}

Group 1=DTaP-IPV-Hep B-PRP-T at 2, 4, 6 months

Group 2=DTaP-IPV-Hep B//PRP-T at 2, 4, 6 months

Data are \% participants $(95 \% \mathrm{Cl})$ or geometric mean concentration $(\mathrm{GMC})$ (calculated according to the number of participants available for the endpoint); $\mathrm{N}=$ number of participants in the per protocol analysis set; $\mathrm{NC}=$ not calculated (as not primary objective

Table 1: Summary of seroprotection rates and geometric mean concentrations (per protocol analysis set).

'Grade 1, 2, and 3 pains were defined as 'minor reaction when injection site is touched,' 'cries and protests when injection site is touched,' and 'cries when injected limb is moved or the movement of the injected limb is reduced.' For erythema and swelling, a diameter of $<2.5 \mathrm{~cm}$ was assessed as Grade 1 , from 2.5 to $<5 \mathrm{~cm}$ as Grade 2 and $\geq 5 \mathrm{~cm}$ as Grade 3. Grade 1, 2, and 3 fever were defined as temperature $\geq 38.0^{\circ} \mathrm{C}-\leq 38.5^{\circ} \mathrm{C},>38.5^{\circ} \mathrm{C}-\leq 39.0^{\circ} \mathrm{C}$, and $>39.5^{\circ} \mathrm{C}$, respectively. Other systemic symptoms were defined as: vomiting (Grade 1-Grade 2, 1 to 5 episodes/day; Grade 3, $\geq 6$ episodes /day) abnormal crying (Grade 1-Grade 2, $\leq 3$ hours; Grade 3, >3 hours), drowsiness, (Grade 1-Grade 2, unusually sleepy; Grade 3, sleepy most of the time) loss of appetite (Grade 1-Grade 2, missed 1 to 2 meals; Grade 3 , missed $\geq 3$ meals) and irritability (Grade 1-Grade 2, easily consolable or needs increased attention; Grade 3, inconsolable). 
Citation: Lanata C, Zambrano B, Ecker L, Amemiya I, Gil A, et al. (2012) Immunogenicity and Safety of a Fully Liquid DTaP-IPV-Hep B-PRP-T Vaccine at 2-4-6 Months of Age in Peru. J Vaccines Vaccin 3:128. doi:10.4172/2157-7560.1000128

\section{Results}

\section{Participants studied}

A total of 266 participants were included in the study as planned, although three participants failed to provide a baseline blood sample and so were withdrawn from the study before receiving the first vaccination. A total of 263 participants received three primary series vaccinations (132 in Group 1 and 131 in Group 2) and were included in the ITT and Safety Analysis Sets. One participant in Group 2 was excluded from the PP analysis set as no post-vaccination blood sample was available, leaving 132 (Group 1) and 130 (Group 2) participants in the PP analysis set and satisfying the sample size power calculation for the primary objective. The participant disposition is summarized in Figure 1.

There were no clinically important differences in demographic characteristics between the two groups.

\section{Immunogenicity}

The observed SP rates 1 month after the third dose were high antiHep B, anti-PRP and anti-D in both groups (Table 1). For anti-Hep B, non-inferiority for SP (concentration $\geq 10 \mathrm{mIU} / \mathrm{mL}$ ) was demonstrated for Group 1 compared to Group 2 (99.2\% in Group 1 and $100.0 \%$ in Group 2) as the lower bound of the $95 \%$ CI for the difference was -4.17 (above the pre-defined lower limit of -10) (Table 1).

After the third dose, at least $99.2 \%$ and $83.8 \%$ of participants had anti-PRP $\geq 0.15$ and $1.0 \mu \mathrm{g} / \mathrm{mL}$ in each group. The SP rate was at least 95.5\% for anti-D $\geq 0.01 \mathrm{IU} / \mathrm{mL}$, and at least $93.9 \%$ for anti-Hep B $\geq 100$ $\mathrm{mIU} / \mathrm{mL}$ in each group.

As for SP rates, anti-Hep B, anti-PRP and anti-D GMCs 1 month after the third primary series vaccination were descriptively similar for each group (Table 1). In addition, anti-D SP rates and GMC were high prior to the first vaccination, and the anti-D antibody GMC decreased from pre-first dose to post-third dose.

The results for the ITT analysis set (not presented) supported those for the PP analysis set.

\section{Safety and tolerability}

No immediate adverse events (i.e. those occurring in the 30 minutes after vaccination) were reported in either group. Solicited injection site and systemic reactions (all grades and Grade 3) are summarized in Table 2. Overall the frequency of each solicited reaction was similar between groups and there was no difference between groups in the frequency of Grade 3 reactions for any solicited injection site or solicited systemic reaction.

The frequency of unsolicited events (systemic and injection site combined) within 7 days after any vaccination was similar in each group $(56.1 \%$ of participants experiencing at least one unsolicited adverse event in Group 1 and 61.8\% in Group 2) and to Day 30 (81.8\% of participants in Group 1 and $87.8 \%$ in Group 2). The most common unsolicited events were nasopharyngitis and abdominal pain, and most were Grade 1 or 2 in intensity, occurred in the 7 days after vaccination, resolved spontaneously, and were not considered to be related to the vaccination.

In Groups 1 and 2 respectively, $3(2.3 \%)$ and $2(1.5 \%)$ participants experienced an SAE in the period up to 30 days after the third vaccination. These were a hepatic cyst (Group 2), cellulitis (Group 1), viral pneumonia (Group 1), and bronchial obstruction (Group 1 and Group 2), and in all cases the participant recovered without sequelae. None of these SAEs was considered by the Investigators to be related to the vaccination.

No participant died during the study and none was withdrawn due to an adverse event.

\section{Discussion}

The primary objective of the study was met, namely to demonstrate non-inferiority in terms of SP rate $(\geq 10 \mathrm{mIU} / \mathrm{mL})$ for the new Hep $\mathrm{B}$ component of the investigational DTaP-IPV-Hep B-PRP-T fully liquid vaccine compared to the licensed DTaP-IPV-Hep B//PRP-T comparator. Furthermore, the post primary series GMCs and the percentage of participants with a concentration $\geq 100 \mathrm{mIU} / \mathrm{mL}$,

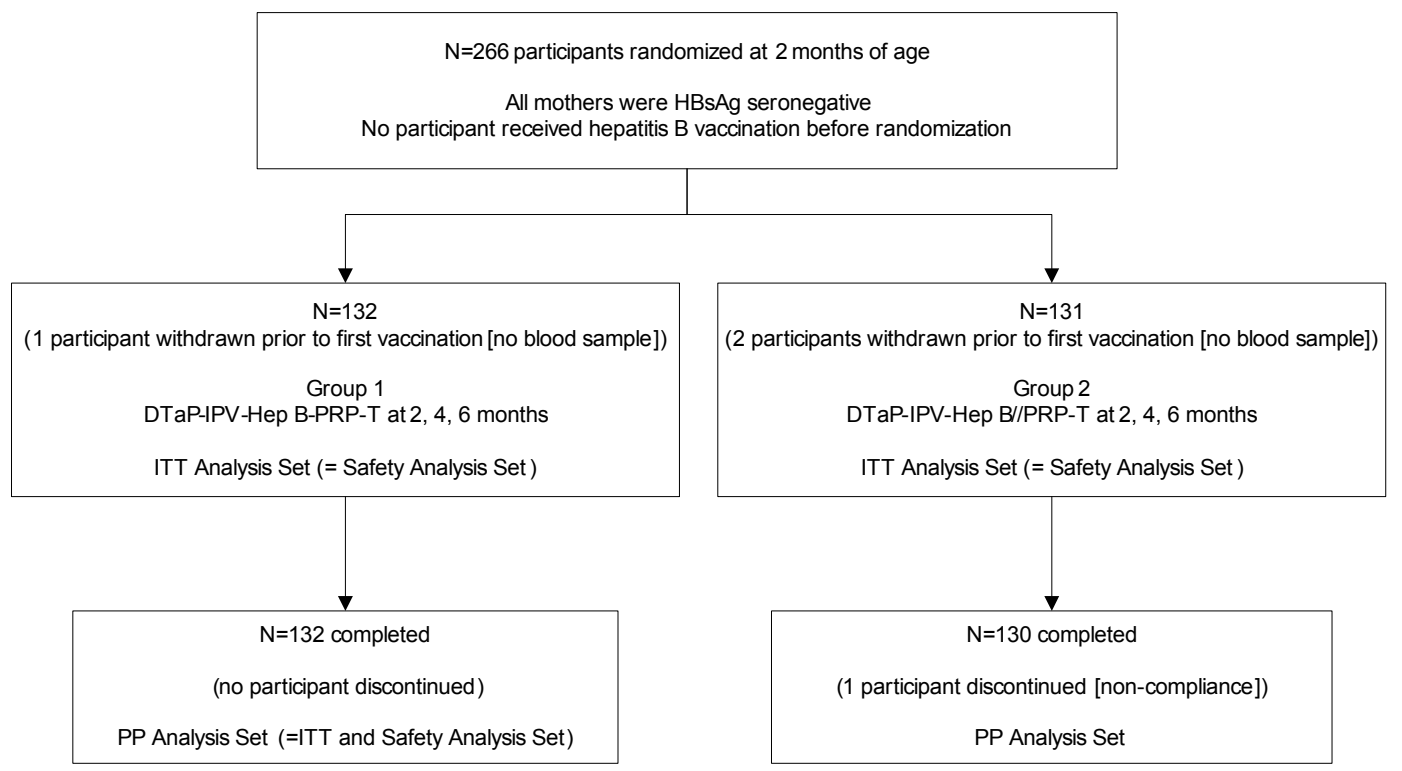

Figure 1: Participant disposition. 


\begin{tabular}{|c|c|c|c|}
\hline Reaction & Severity & Group $1(\mathrm{~N}=132)$ & Group $2(\mathrm{~N}=131)$ \\
\hline \multicolumn{4}{|c|}{ Injection Site Reactions: } \\
\hline \multirow[t]{2}{*}{ Pain } & Any & $77.3(69.2 ; 84.1)$ & $77.1(68.9 ; 84.0)$ \\
\hline & Grade 3 & $7.6(3.7 ; 13.5)$ & $4.6(1.7 ; 9.7)$ \\
\hline \multirow[t]{2}{*}{ Erythema } & Any & $59.1(50.2 ; 67.6)$ & $50.4(41.5 ; 59.2)$ \\
\hline & Grade 3 & $2.3(0.5 ; 6.5)$ & $3.8(1.3 ; 8.7)$ \\
\hline \multirow[t]{2}{*}{ Swelling } & Any & $40.9(32.4 ; 49.8)$ & $39.7(31.3 ; 48.6)$ \\
\hline & Grade 3 & $2.3(0.5 ; 6.5)$ & $1.5(0.2 ; 5.4)$ \\
\hline \multicolumn{4}{|c|}{ Systemic Reactions: } \\
\hline \multirow[t]{2}{*}{ Pyrexia } & Any & $28.0(20.6 ; 36.5)$ & $27.5(20.0 ; 36.0)$ \\
\hline & Grade 3 & $0.0(0.0 ; 2.8)$ & $2.3(0.5 ; 6.5)$ \\
\hline \multirow[t]{2}{*}{ Vomiting } & Any & $22.0(15.2 ; 30.0)$ & $24.4(17.3 ; 32.7)$ \\
\hline & Grade 3 & $0.0(0.0 ; 2.8)$ & $0.0(0.0 ; 2.8)$ \\
\hline \multirow[t]{2}{*}{ Crying } & Any & $75.8(67.5 ; 82.8)$ & $71.0(62.4 ; 78.6)$ \\
\hline & Grade 3 & $0.8(0.0 ; 4.1)$ & $0.8(0.0 ; 4.2)$ \\
\hline \multirow[t]{2}{*}{ Somnolence } & Any & $55.3(46.4 ; 64.0)$ & $62.6(53.7 ; 70.9)$ \\
\hline & Grade 3 & $1.5(0.2 ; 5.4)$ & $1.5(0.2 ; 5.4)$ \\
\hline \multirow[t]{2}{*}{ Anorexia } & Any & $40.9(32.4 ; 49.8)$ & $44.3(35.6 ; 53.2)$ \\
\hline & Grade 3 & $0.0(0.0 ; 2.8)$ & $0.8(0.0 ; 4.2)$ \\
\hline \multirow[t]{2}{*}{ Irritability } & Any & $75.8(67.5 ; 82.8)$ & $74.8(66.5 ; 82.0)$ \\
\hline & Grade 3 & $1.5(0.2 ; 5.4)$ & $0.8(0.0 ; 4.2)$ \\
\hline
\end{tabular}

Group 1=DTaP-IPV-Hep B-PRP-T at 2, 4, 6 months

Group 2=DTaP-IPV-Hep B//PRP-T at 2, 4, 6 months

Data are $\%$ participants $(95 \% \mathrm{Cl}$ ) (calculated according to the number of participants available for the endpoint) $\mathrm{N}=$ number of participants in the safety analysis set

Table 2: Summary of solicited injection site and systemic adverse reactions occurring within 7 days after any dose of vaccine (safety analysis set).

indicative of long-term protection, were high and similar in each group. These Hep B data confirm earlier data for both the investigational hexavalent vaccine in a 2, 4, 6 month schedule in Argentina [8] as well as those following administration of a monovalent Hep B vaccine or the comparator hexavalent vaccine using the same schedule [15].

The Hep B component of the investigational vaccine is of particular interest as it is a new antigen, specifically developed for inclusion in the new hexavalent vaccine. The other antigens have well established immunogenicity and safety profiles, being included in other AcXim family vaccines such as Pentaxim ${ }^{\mathrm{TM}} /$ Pentavac $^{\mathrm{TM}}$ and Tetraxim $^{\mathrm{TM}} /$ Tetravac $^{\mathrm{TM}}$. The immunogenicity of these antigens has also been described following administration of the new hexavalent vaccine in earlier clinical studies [6-8] as well as (with the exception of Hep B) after the administration of Pentaxim ${ }^{\mathrm{TM}} /$ Pentavac $^{\mathrm{TM}}[10]$.

In order to accurately assess the immunogenicity of the new Hep B antigen, only mothers who were seronegative to Hep B were included and no Hep B vaccine was administered at birth or in the 2 months prior to entry into the clinical study (the birth dose specified in the Peruvian national vaccination calendar was postponed for the purposes of the study). A birth dose of Hep B vaccine is particularly important for preventing the development of the disease in babies at risk of vertical transmission, i.e. those born to seropositive mothers [16,17]: as only babies with no risk of vertical transmission were selected, the omission of a Hep B birth dose posed no risk to those included in the study.

Anti-PRP antibodies were assessed since this antigen is the only lyophilized antigen in Pentaxim ${ }^{\mathrm{TM}} /$ Pentavac $^{\mathrm{TM}}$, but is non-lyophilized when included as part of a fully liquid formulation in the investigational hexavalent vaccine. Previous studies with Pentaxim ${ }^{\mathrm{TM}} /$ Pentavac $^{\mathrm{TM}}$ (which includes the same DTaP-IPV//PRP-T backbone as the investigational vaccine) have shown that the percentage of children seroprotected $(\geq 0.15 \mu \mathrm{g} / \mathrm{mL})$ is similar to those receiving monovalent Hib vaccine,

although the response in terms of anti-PRP $\geq 1.0 \mu \mathrm{g} / \mathrm{mL}$ is more variable than for the monovalent vaccine. However, the quality of the immune responses have been shown to be the same $[18,19]$, particularly in terms of long-term epidemiology [20,21]. We also focused on this antigen since some previous reports have suggested the possibility of a reduced PRP response following administration when combined with aPcontaining vaccines, leading to lower GMCs compared to monovalent Hib vaccines [22-27]. In our study, the anti-PRP antibody GMC was in fact slightly higher for the fully liquid investigational vaccine that for the lyophilised comparator, although there was no difference between groups in the percentage of participants with a concentration $\geq 0.15 \mu \mathrm{g} /$ $\mathrm{mL}$ or $\geq 1.0 \mu \mathrm{g} / \mathrm{mL}$. For each threshold, the response was comparable to monovalent Hib vaccine administration at 2, 4, 6 months of age [19], confirming no reduction in the PRP response for the investigational hexavalent vaccine.

The measurement of anti-D antibodies before and after the primary series was to document the putative presence of residual maternal antibodies post-natally and their effect on the post-natal response to vaccination. Our data show high SP rates against diphtheria prior to the first vaccination, resulting from the passive, maternally-acquired immunity. Although the percentage of participants with antidiphtheria antibodies $\geq 0.01 \mathrm{IU} / \mathrm{mL}$ was higher after the primary series than before the first vaccination, our data show slightly reduced GMCs in both groups and a slightly reduced percentage of participants with a concentration $\geq 0.1 \mathrm{IU} / \mathrm{mL}$ after the primary series. This is a well documented phenomenon, having previously been described for the investigational vaccine [8] as well as for other pediatric diphtheriacontaining vaccines [28-30].

The safety of the investigational vaccine was similar to that of the comparator vaccine, and confirmed its good safety profile documented in previous clinical studies [6-8].

\section{Conclusions}

The investigational DTaP-IPV-Hep B-PRP-T vaccine $\left(\right.$ Hexaxim $\left.^{\mathrm{TM}}\right)$ demonstrated high immunogenicity for the new Hep B antigen and for the PRP antigen (as part of a fully liquid, hexavalent presentation) that was similar to a licensed hexavalent comparator vaccine (with a lyophilized PRP antigen). Additionally, the assessment of anti-D antibodies confirmed the presence of maternal antibodies prior to the first dose and their inhibitory influence on the response to the primary vaccination series in terms of GMC (although in terms of the percentage of participants with a titer $\geq 0.01 \mathrm{mIU} / \mathrm{mL}$, the post-vaccination SP rate was higher than pre-vaccination). The investigational vaccine demonstrated a good safety profile, consistent with that demonstrated in previous clinical studies with the same vaccine. The expanded use of such an aP-IPV-containing hexavalent vaccine could contribute to further improvement in vaccine coverage and an associated reduction in the burden of these six childhood diseases.

\section{Source of Financial Support}

This study was done with the financial support of Sanofi Pasteur, Lyon, France.

The results of this study were presented at the Sociedad Latinoamericana de Infectología Pediátrica (SLIPE), Dominican Republic, 25-28 May 2011.

\section{Acknowledgements}

We would like to acknowledge all the study staff who contributed to the study conduct and to all study participants and their parents/legal guardians.

In addition, we would like to thank the members of an independent data 
monitoring committee (IDMC) for periodic review of safety data during this study: Dr Edwin Asturias (Chairman and voting member), Prof Fred Zepp (voting member) Dr Alain Leizorovicz (voting member), Dr Rosanna Lagos (voting member), and Dr Nadine Bossard (non-voting member).

We would also like to thank Hector Verástegui of the Instituto de Investigación Nutricional for data management support and data checking, Dr Sandrine LentschGraf (Hexaxim ${ }^{\text {Th }}$ Project Leader) for her valuable input, Mrs Siham B'Chir for the statistical analyses, and $\mathrm{Dr}$ Andrew Lane for assistance in the preparation and development of the manuscript in accordance with the European Medical Writers Association guidelines and Good Publication Practice. SL-G, SB and AL are employees of Sanofi Pasteur.

\section{Conflicts of Interest}

This study was sponsored by Sanofi Pasteur who provided funding to the Instituto de Investigación Nutricional for this purpose. CL, LE, IA, and AG are employees of the Instituto de Investigación Nutricional but received no direct payment from Sanofi Pasteur. ESL and BZ are employees of Sanofi Pasteur. None of the IDMC members was employed by, or received payment from, Sanofi Pasteur (other than expenses).

\section{References}

1. Diminsky D, Schirmbeck R, Reimann J, Barenholz Y (1997) Comparison between hepatitis $\mathrm{B}$ surface antigen $(\mathrm{HBsAg})$ particles derived from mammalian cells $(\mathrm{CHO})$ and yeast cells (Hansenula polymorpha): composition, structure and immunogenicity. Vaccine 15: 637-647.

2. Greiner VJ, Egelé C, Oncul S, Ronzon F, Manin C, et al. (2010) Characterization of the lipid and protein organization in HBsAg viral particles by steady-state and time-resolved fluorescence spectroscopy. Biochimie 92: 994-1002.

3. Milhiet PE, Dosset P, Godefroy C, Le Grimellec C, Guigner JM, et al. (2011) Nanoscale topography of hepatitis B antigen particles by atomic force microscopy. Biochimie 93: 254-259.

4. World Health Organization ((2010)) United Nations pre-qualified vaccines (accessed 04 August 2011)

5. Tregnaghi MW, Voelker R, Santos-Lima E, Zambrano B (2010) Immunogenicity and safety of a novel yeast Hansenula polymorpha-derived recombinan Hepatitis B candidate vaccine in healthy adolescents and adults aged 10-45 years. Vaccine 28: 3595-3601.

6. Kosalaraska P, Thisyakorn U, Benjaponpitak S, Chokephaibulkit K, SantosLima E (2011) Immunogenicity and safety study of a new DTaP-IPV-Hep B-PRP-T combined vaccine compared to a licensed DTaP-IPV-Hep B//PRP-T comparator, both concomitantly administered with a 7 -valent pneumococcal conjugate vaccine at 2, 4, 6 months of age in Thai infants. Int $\mathrm{J}$ Infect Dis 15 : e249-256.

7. Madhi SA, Mitha I, Cutland C, Groome M, Santos-Lima E (2011) Immunogenicity and Safety of an Investigational Fully Liquid Hexavalent Combination Vaccine Versus Licensed Combination Vaccines at 6, 10, and 14 Weeks of Age in Healthy South African Infants. Pediatr Infect Dis J 30: e68-74.

8. Tregnaghi MW, Zambrano B, Santos-Lima E (2011) Immunogenicity and safety of an investigational hexavalent diptheria-tetanus-acellular pertussis-inactivated poliovirus-hepatitis B-Haemophilus influenzae B conjugate combined vaccine in healthy 2-, 4-, and 6-month-old Argentinean infants. Pediatr Infect Dis J 30: e88-96.

9. Decker M, Bogaerts H, Edwards K. Combination vaccines (2008) In: Vaccines, (5thedn). Plotkin S, Orenstein W, Offit PA (Eds), Saunders and Co., PA, USA 1069-1101.

10. Plotkin SA, Liese J, Madhi SA, Ortiz E (2011) A DTaP-IPV//PRP approximately $T$ vaccine (Pentaxim): a review of 16 years' clinical experience. Expert Rev Vaccines 10: 981-1005

11. Vidor E, Plotkin SA (2008) Immunogenicity of a two-component (PT \& FHA) acellular pertussis vaccine in various combinations. Hum Vaccin 4: 328-340.

12. Newcombe RG (1998) Interval estimation for the difference between independent proportions: comparison of eleven methods. Stat Med 17: 873890

13. Newcombe RG (1998) Two-sided confidence intervals for the single proportion comparison of seven methods. Stat Med 17: 857-872.
14. Farrington CP, Manning G (1990) Test statistics and sample size formulae for comparative binomial trials with null hypothesis of non-zero risk difference or non-unity relative risk. Stat Med 9: 1447-1454.

15. Arístegui J, Dal-Ré R, Díez-Delgado J, Marés J, Casanovas JM, et al. (2003) Comparison of the reactogenicity and immunogenicity of a combined diphtheria tetanus, acellular pertussis, hepatitis B, inactivated polio (DTPa-HBV-IPV) vaccine, mixed with the Haemophilus influenzae type $b$ (Hib) conjugate vaccine and administered as a single injection, with the DTPa-IPV/Hib and hepatitis B vaccines administered in two simultaneous injections to infants at 2, 4 and 6 months of age. Vaccine 21: 3593-3600.

16. Thisyakorn U, Montellano M, Lane A (2011) Routine newborn hepatitis $B$ immunization: a review of schedules. Inf Dis Clin Prac 19: 326-331.

17. World Health Organisation (2009) Hepatitis B position paper. Weekly Epidemio Rec 84: 405-420.

18. Eskola J, Ward J, Dagan R, Goldblatt D, Zepp F, et al. (1999) Combined vaccination of Haemophilus influenzae type $b$ conjugate and diphtheriatetanus-pertussis containing acellular pertussis. Lancet 354: 2063-2068.

19. Hoppenbrouwers K, Kanra G, Roelants M, Ceyhan M, Vandermeulen C, et al. (1999) Priming effect, immunogenicity and safety of an Haemophilus influenzae type b-tetanus toxoid conjugate (PRP-T) and diphtheria-tetanus-acellular pertussis (DTaP) combination vaccine administered to infants in Belgium and Turkey. Vaccine 17: 875-886.

20. Greenberg DP, Doemland M, Bettinger JA, Scheifele DW, Halperin SA, et al (2009) Epidemiology of pertussis and Haemophilus influenzae type b disease in Canada with exclusive use of a diphtheria-tetanus-acellular pertussisinactivated poliovirus-Haemophilus influenzae type $b$ pediatric combination vaccine and an adolescent-adult tetanus-diphtheria-acellular pertussis vaccine: implications for disease prevention in the United States. Pediatr Infect Dis $J$ 28: 521-528.

21. Schmitt HJ, von Kries R, Hassenpflug B, Hermann M, Siedler A, et al. (2001) Haemophilus influenzae type $b$ disease: impact and effectiveness of diphtheriatetanus toxoids-acellular pertussis (-inactivated poliovirus)/ $\mathrm{H}$. influenzae type $b$ combination vaccines. Pediatr Infect Dis J 20: 767-774.

22. Lagos R, Kotloff K, Hoffenbach A, San Martin O, Abrego P, et al. (1998) Clinica acceptability and immunogenicity of a pentavalent parenteral combination vaccine containing diphtheria, tetanus, acellular pertussis, inactivated poliomyelitis and Haemophilus influenzae type b conjugate antigens in twofour- and six-month-old Chilean infants. Pediatr Infect Dis J 17: 294-304

23. Bell F, Heath P, Shackley F, MacLennan J, Shearstone N, et al. (1998) Effect of combination with an acellular pertussis, diphtheria, tetanus vaccine on antibody response to Hib vaccine (PRP-T). Vaccine 16: 637-642.

24. Eskola J, Olander RM, Hovi T, Litmanen L, Peltola S, et al. (1996) Randomised trial of the effect of co-administration with acellular pertussis DTP vaccine on immunogenicity of Haemophilus influenzae type b conjugate vaccine. Lance 348: 1688-1692.

25. Halperin SA, Tapiero B, Diaz-Mitoma F, Law BJ, Hoffenbach A, et al. (2009) Safety and immunogenicity of a hexavalent diphtheria-tetanus-acellula pertussis-inactivated poliovirus-Haemophilus influenzae $b$ conjugate-hepatitis $B$ vaccine at 2, 3, 4, and 12-14 months of age. Vaccine 27: 2540-2547.

26. Pichichero ME, Passador S (1997) Administration of combined diphtheria and tetanus toxoids and pertussis vaccine, hepatitis B vaccine, and Haemophilus influenzae type $b(\mathrm{Hib})$ vaccine to infants and response to a booster dose of $\mathrm{Hib}$ conjugate vaccine. Clin Infect Dis 25: 1378-1384.

27. Poolman J, Kaufhold A, De Grave D, Goldblatt D (2001) Clinical relevance of lower Hib response in DTPa-based combination vaccines. Vaccine 19: 2280 2285.

28. Gall SA (2005) Maternal immunization to protect the mother and neonate Expert Rev Vaccines 4: 813-818.

29. Gall SA, Myers J, Pichichero M (2011) Maternal immunization with tetanusdiphtheria-pertussis vaccine: effect on maternal and neonatal serum antibody levels. Am J Obstet Gynecol 204: 334-335.

30. Vitek CR, Wharton M. Diphtheria Toxoid (2008) In: Vaccines, (5thedn). Plotkin SA, Orenstein WA, Offit PA (Eds), Saunders and Co., PA, USA 139-155. 